\title{
Photon-exchange effects on photon-pair transmission
}

\author{
K.J. Resch, G.G. Lapaire, J.S. Lundeen, J.E. Sipe, and A.M. Steinberg \\ Department of Physics, University of Toronto \\ 60 St. George Street, Toronto ON M5S $1 A 7$ \\ CANADA
}

\begin{abstract}
It has been proposed that photon-exchange effects in atom-photon interactions could lead to greatly enhanced optical nonlinearities. These might have widespread application (e.g. quantum information). Here we demonstrate experimentally that such exchange effects can indeed enhance the probability of real absorption of photon pairs. Using nonclassical pairs of photons with variable time separation, we observe a maximum suppression of pair transmission by at least $5 \%$ with respect to the result for uncorrelated photons.
\end{abstract}


Exchange effects play a rich and central role in quantum physics, and are linked with phenomena as diverse as the Pauli exclusion principle and Bose condensation. For both fermions and bosons they result in a modification of the effect of inter-particle interaction from what would be predicted for distinguishable particles, and can often be thought of as leading to a new effective interaction between indistinguishable particles. Photons are bosons, and are usually thought to interact with each other, via their coupling with material media, very weakly. For example, the usual Kerr nonlinearity, which is well-known to grow linearly with the atom density in the interaction region [1] rarely produces phase shifts larger than $10^{-10}$ for a pair of photons. This can be enhanced using schemes employing cavity QED 2], electromagnetically-induced transparency and slow light 3], or interference-based nonlinearities [4]. However, the technical difficulties that plague these protocols have led many who seek an effective photon-photon interaction for applications in quantum computing and information processing to turn instead to the use of linear optics and conditional detection [5, 6] to simulate such an effect. Could an exchange effect instead be leveraged to lead to an effective photon nonlinearity at the few photon level?

Six years ago Jim Franson 7, 8] argued that indeed photon-exchange interactions in an atomic system might give rise to a very strong effective nonlinearity. The process requires a pair of photons at frequencies $\omega_{1}$ and $\omega_{2}$, and a pair of atoms $A$ and $B$. Exchange would occur when atom $A$ nonresonantly 'absorbs' photon 1 and emits photon 2 , while atom $B$ absorbs photon 2 and emits photon 1 . Franson predicted that this exchange-based nonlinear effect would grow as the square of the atom density and could be significant at the two-photon level in a sufficiently dense sample. This proposed effect has been the subject of significant controversy [9], and has not yet been verified in the laboratory.

In this letter we present both a theoretical motivation and experimental realization of a simpler photon-exchange effect, one that involves real transitions in matter rather than virtual transitions. Consider the two-photon absorption probability for like-polarized photons travelling in the same direction in a one-dimensional system. This can easily be calculated in perturbation theory. We consider a two-photon state initially described as:

$$
|A, B\rangle=\mathcal{N}(A, B) \iint d \omega d \omega^{\prime} f_{A}(\omega) f_{B}\left(\omega^{\prime}\right) a^{\dagger}(\omega) a^{\dagger}\left(\omega^{\prime}\right)|0\rangle,
$$

where $a^{\dagger}(\omega)$ is a raising operator for a photon of frequency $\omega, f_{A(B)}(\omega)$ is the frequency amplitude function for an individual photon $A(B)$, and $\mathcal{N}(A, B)=\left(1+\left|\int f_{A}^{*}(\omega) f_{B}(\omega) d \omega\right|^{2}\right)^{-1 / 2}$ is a normalization constant, $f_{A(B)}(\omega)$ are two amplitude functions for frequency $\omega$, and $a^{\dagger}(\omega)$ is the raising operator for a photon of frequency $\omega$. Under these conditions, the probability of absorbing both photons, $P_{A B}$, can be expressed in terms of the single-photon absorption probabilities $P_{A}$ and $P_{B}$ :

$$
P_{A B}=P_{A} P_{B}\left(\frac{1+\xi_{A B}}{1+v_{A B}}\right)
$$

where $v_{A B}$ is the square of the overlap integral, $\left|\int d \omega f_{A}^{*}(\omega) f_{B}(\omega)\right|^{2} \leqslant 1$, and

$$
\xi_{A B}=\frac{\left|\int d \omega g(\omega) f_{A}^{*}(\omega) f_{B}(\omega)\right|^{2}}{\left[\int d \omega g(\omega)\left|f_{A}(\omega)\right|^{2}\right]\left[\int d \omega g(\omega)\left|f_{B}(\omega)\right|^{2}\right]},
$$

where $g(\omega)$ is the absorption spectrum of the medium. For independent absorption events we expect $P_{A B}=P_{A} P_{B}$; therefore correlated absorption probabilities come from cases where $v_{A B} \neq \xi_{A B}$. Such a case can be set up if the two photons are separated in time, but pass through a medium with a narrow absorption feature of width, $\Delta \omega_{a}$. Since the photons do not overlap, $v_{A B} \approx 0$. Provided that the absorber has a coherence time, $\tau \equiv 1 / \Delta \omega_{a}$, longer than the delay between the photons, we may have $\xi_{A B} \neq 0$. In other words, if the photons are distinguishable before absorption, but become (mostly) indistinguishable if absorbed, we may have $P_{A B}>P_{A} P_{B}$ - an enhancement of the two-photon absorption. In cases where the photon delays are much longer than the coherence time of the absorber, or when the photons are perfectly overlapped, $P_{A B}=P_{A} P_{B}$, as expected in the absence of any nonlinear effects.

It is possible to move beyond a perturbative approach with a photon-wavefunction picture, even for two-photon states $|A, B\rangle$ which are not restricted to one-dimension (as in Eq. 1). But in that one-dimensional limit (as in Eq. 1), in the absence of any absorber the coincidence detection rate of two ideal detectors at positions $z_{1}, z_{2}$ and at times $t_{1}, t_{2}$ is proportional to,

$$
w^{(2)}\left(z_{1}, z_{2}, t_{1}, t_{2}\right)=|\mathcal{N}(A, B)|^{2}\left[\begin{array}{c}
\left|\psi_{A}\left(z_{1}, t_{1}\right)\right|^{2}\left|\psi_{B}\left(z_{2}, t_{2}\right)\right|^{2}+\left|\psi_{A}\left(z_{2}, t_{2}\right)\right|^{2}\left|\psi_{B}\left(z_{1}, t_{1}\right)\right|^{2} \\
+\psi_{A}^{*}\left(z_{1}, t_{1}\right) \psi_{B}\left(z_{1}, t_{1}\right) \psi_{B}^{*}\left(z_{2}, t_{2}\right) \psi_{A}\left(z_{2}, t_{2}\right) \\
+\psi_{A}^{*}\left(z_{2}, t_{2}\right) \psi_{B}\left(z_{2}, t_{2}\right) \psi_{B}^{*}\left(z_{1}, t_{1}\right) \psi_{A}\left(z_{1}, t_{1}\right) .
\end{array}\right] .
$$


The first-order photon wavefunctions, $\psi_{A(B)}(z, t)$, satisfy $E^{+}(z, t)|A(B)\rangle=\psi_{A(B)}(z, t)|0\rangle$, where $E^{+}(z, t)$ is the positive frequency component of the electric field operator, and $|A(B)\rangle=\int d \omega f_{A(B)}(\omega) a^{\dagger}(\omega)|0\rangle$ is a single-photon state. The last two terms on the right-hand side of Eq. [ are the exchange terms. In the absence of these terms, the coincidence detection rate is proportional to the sum of the product of the individual photon detection rates at either detector, which is characteristic of independent detection events. Applying this wavefunction description to a Hong-Ou-Mandel interferometer [10], one can show that the Hong-Ou-Mandel dip is simply a manifestation of photon exchange. Considering now the presence of an absorbing medium, the photon wavefunctions allow one to see the underlying physics in which, in this case, destructive interference occurs between the detection events. The wavefunctions, perhaps initially nonoverlapping before passage throught the absorbing medium, may no longer be after their passage through it. Thus there can be interference effects in the subsequent detection, and a corresponding reduction in the two-photon transmission probability below the uncorrelated-absorption prediction. This is the experimental signature we seek.

To investigate the exchange-induced suppression of two-photon transmission we used the basic system sketched in Fig. 1. There are essentially three parts to both the experiment and the theory: state preparation, evolution, and detection. The quantum state of interest is a normalized two-photon state where both photons have the same polarization, same spectrum and a variable inter-photon time delay. This state evolves as it passes through an absorptive medium which has some resonant absorption feature in the centre of the photon spectrum. Finally, the photon pairs that emerge from the medium are counted.

The two-photon states were created in the setup shown in Fig. 2. The setup is similar to a polarization-based Hong-Ou-Mandel (HOM) interferometer [10, 11] whereby the desired two-photon state is post-selected through the detection of a photon pair. Specifically, we used a collinear type-II phase-matched parametric down-conversion source (0.1-mm thick BBO) pumped by the second harmonic of a Ti:Sapphire laser. The second harmonic was centred at $405 \mathrm{~nm}$ (with a bandwidth of $7 \mathrm{~nm}$ FWHM) and created pairs of photons each with centre wavelengths of $810 \mathrm{~nm}$. The photon pairs exit from the source such that one has vertical polarization and the other horizontal. We control the relative time delay between the photons by passing them through a modified Babinet compensator. After the variable delay, a polarizer is placed in the photons' path at $45^{\circ}$. With the polarizer in the system, any photon pairs transmitted through this polarizer are polarized at $45^{\circ}$, with a time separation determined by the Babinet. This serves as the source of two-photon states required by the theoretical work. From HOM interference [10], we expect an increase in the number of photon pairs created near zero delay as the photons tend to pair up. We measure the rate of photon-pair production for each delay, in the absence of any absorber, and use this to normalize our subsequent absorption experiment.

The theory also requires an absorber with an absorption feature narrower than the bandwidth of the photon pairs. From our down-conversion source, we obtain photon pairs with a FWHM power spectrum of over 100nm. We used a dielectric interference filter (CVI F10-810-4-1.00) as an effective absorbing medium. The back of the filter was blacked-out, and so any light transmitted through it was discarded. The reflectivity of the filter shows a $10 \mathrm{~nm}$ wide dip centred at $810 \mathrm{~nm}$. The reflected light rhus plays the role of light nominally transmitted through an absorber with a $10 \mathrm{~nm}$ absorption feature, and we refer to it as such below. It should be noted that a gaseous atomic sample could be used if one used a narrower bandwidth down-conversion source [12].

We began by examining the reflection from a broadband dielectric mirror in order to measure the number of reflected photon pairs as a function of delay using a cascaded pair of SPCMs (Perkin Elmer SPCM-AQR-13) 13]. This data set shows any changes in the efficiency of two-photon state production, which will be divided out. Then the interference filter (with the blacked-out back) is placed directly in front of a broadband dielectric mirror. With the filter in place, we measured the number of remaining photon pairs in the reflection. We monitored the detectors' singles rates and their coincidence rate in the experiment.

Fig. 3 shows the rates of photon-pair detection (solid circles) and singles rate (small open diamonds) at one of the detectors as a function of the time delay between the photons. Fig. 3a shows the data taken with the broadband mirror in place. It clearly shows that the two-photon state preparation becomes much more efficient at zero delay. This increased pairing is due to HOM interference [10]. In our experimental results, the rate of photon pair production at zero delay is $55 \%$ larger than that at large time delays, whereas perfect HOM interference leads to a doubling of the rate. As one would expect from a HOM interferometer with low collection or detection efficiencies, the singles rate is featureless at the $1 \%$ level as a function of the time delay between the photons [14]. Fitting the data in Fig. $3 \mathrm{a}$ under the assumption of identical gaussian power spectra for the two photons yielded a FWHM of $129 \mathrm{~nm}$.

Fig. 3b shows the data taken with the interference filter in place. The most striking difference from the previous figure is the drop in the number of photon pairs detected at a delay of approximately $\pm 10 \mathrm{fs}$. There is a second, more subtle, difference in that the number of photon pairs at zero delay is enhanced by only $42 \%$ over the rate at large time delays with the filter in place. The singles rate in Fig. $3 \mathrm{~b}$ also shows no dependence on the time delay at 
the $1 \%$ level.

The ratio of the data in Fig. 3b to Fig. 3a is shown in Fig. 4 as solid circles. This ratio normalizes the data for changes in the production efficiency of two-photon states and shows the photon pair reflection probability given a two-photon input. To reduce the noise on the data points a 5-point average was taken. We compare the observed rate of photon pair transmission at $\pm 10 \mathrm{fs}$ to long- and zero-time delays. The normalized photon-pair detection ratio at $-10 \mathrm{fs}$ is $15 \%$ less than that at long delays and $5 \%$ less than that at zero; the ratio at +10 fs is about $17 \%$ less than at long delays and $7 \%$ less than that at zero. The asymmetry in the data may be due to dispersion effects in the nonlinear crystal [15]. Fig. 4 also shows the theoretical predictions for the coincidence rate ratio as a function of the time delay between the photons in two different regimes using gaussian spectra and a gaussian absorption feature. The two different curves correspond to photon spectra that are initially uncorrelated (solid curve), as in Eq. 1, and where the sum of the photon energies is a constant (dotted curve). The first regime describes the light produced from a down-conversion source with a broadband pump laser after sufficiently narrow bandpass filtering, whereas the second describes down-conversion created by a CW laser [16, 17]. The curves are both scaled to their coincidence rate at very large time delays. The drop in the ratio measured at zero delay which is not predicted by the theory. In our theoretical description, the photons are perfectly indistinguishable at zero time delay. Thus, passing through the absorbing medium can do nothing to change their degree of distinguishability and the rate of photon-pair absorption is that of uncorrelated events. In our experiment, however, the photons are still partially distinguishable even at zero delay due to imperfect spectral and spatial mode-matching. When some light is absorbed by the medium, the overlap between the photon wavefunctions are changed and this leads to an additional effect at zero delay. Photon-exchange terms are responsible for at least the $5 \%$ change in the photon pair transmission through our absorbing media at \pm 10 fs.

In both situations, the enhanced region of photon absorption occurs at approximately $\pm 10 \mathrm{fs}$, in good agreement with experiment. The $5 \%$ suppression in the rate of photon-pair transmission is on the order of that predicted by our theory, but a more accurate comparison will require better data or a more complete theory that includes imperfect spatial- and spectral-mode matching. The shape of our experimental curve is in better agreement with the theoretical curve with no frequency correlations where the transmission reaches its long delay value at about \pm 20 fs. The theory for perfect frequency correlations shows suppressed transmission over much longer times. In our experiment we are actually between these two extreme regimes; our ultrafast-pump laser has a bandwidth of about $7 \mathrm{~nm}$. From preliminary calculations, we have found that by accounting for partial frequency correlations, suppression in photon pair transmission can be enhanced over the case with no correlations without significantly changing the shape of the curve. It is clear from the theory that these correlations can greatly influence the photon-pair transmission probability. Such a striking dependence on these correlations makes this technique useful for measuring them.

In summary, we have observed that photon-exchange effects can give rise to nonlinear behaviour on the pairtransmission probability. Like-polarized photon pairs with a variable inter-particle delay have been shown to exhibit suppressed two-photon transmission through a linearly absorbing medium - an experimental signature of exchange enhancement of photon-pair absorption. This suppression occurs for delays that are longer than the photons' coherence times but shorter than the coherence time of the absorber. Further work is needed to understand the limits on the applicability of such exchange effects to nonlinear optics and quantum information.

This work was funded by Photonics Research Ontario, NSERC, and the U.S. Air Force Office of Scientific Research (F49620-01-1-0468).

[1] See for example A. L. Gaeta and R. W. Boyd, in Atomic, Molecular and Optical Physics Handbook, edited by G. W. F. Drake (American Institute of Physics, Woodbury, New York, 1996) p. 809.

[2] Q. A. Turchette, et al., Phys. Rev. Lett., 75, 4710 (1995); A. Rauschenbeutel, et al., Phys. Rev. Lett., 83, 5166 (1999).

[3] S.E. Harris, J.E. Field, and A. Imamoğlu, Phys. Rev. Lett., 64, 1107 (1990); A. Kasapi, et al., Phys. Rev. Lett., 74, 2447 (1995); S.E. Harris and L.V. Hau, Phys. Rev. Lett., 82, 4611 (1999); L.V. Hau, et al., Nature, 397, 594 (1999); M.M. Kash, et al., Phys. Rev. Lett., 82, 5229 (1999).

[4] K. J. Resch, J. S. Lundeen, and A. M. Steinberg, Phys. Rev. Lett., 87, 123603 (2001); K. J. Resch, J. S. Lundeen, and A. M. Steinberg, Phys. Rev. Lett., 89, 037914 (2002).

[5] E. Knill, R. Laflamme, and G. J. Milburn, Nature, 409, 46 (2001).

[6] T. C. Ralph, et al., Phys. Rev. A, 65, 012314 (2002); T. Rudolph and J.-W. Pan, quant-ph/0108056, (2001).

[7] J. D. Franson, Phys. Rev. Lett., 78, 3852 (1997).

[8] J. D. Franson and T. B. Pittman, Fortschritte der Physik, 46, 697 (1998); J. D. Franson and T. B. Pittman, Phys. Rev. A, 60, 917 (1999); J. D. Franson, Fortschritte der Physik, 48, 1133 (2000).

[9] T. Opatrný and G. Kurizki, Fortschritte der Physik, 48, 1125 (2000); M. Fleischhauer, quant-ph/0006042 (2000). 
[10] C.K. Hong, Z.Y. Ou, and L. Mandel, Phys. Rev. Lett. 59, 2044 (1987).

[11] G. Di Giuseppe, et al., Phys. Rev. A, 56, R21 (1997).

[12] J. H. Shapiro, N. C. Wong, J. Opt. B: Quant. Semiclass. Opt., 2, L1 (2000); Y. J. Lu and Z. Y. Ou, Phys. Rev. A, 62, $033804(2000)$.

[13] Z.Y. Ou, J.-K. Rhee, and L.J. Wang, Phys. Rev. Lett., 83, 959 (1999).

[14] K.J. Resch, J.S. Lundeen, and A.M. Steinberg, Phys. Rev. A, 63, 020102(R) (2001).

[15] M. Atatüre, et al., Phys. Rev. Lett., 83, 1323 (1999).

[16] M. Atatüre, et al., Phys. Rev. A, 66, 023822 (2002).

[17] W.P. Grice and I.A. Walmsley, Phys. Rev. A, 56, 1627 (1997). 
Fig. 1. The system of interest. A two-photon state with a variable time delay between the likepolarized photons impinges on an absorbing medium. The light that passes through the absorption region is detected by a photon-pair detector. The probability of absorbing a photon pair can depend very strongly on the delay.

Fig. 2. The experimental setup. The state preparation is accomplished using the output of a polarization-based HOM interferometer [10, 11]. BBO is a $\beta$-barium borate nonlinear crystal phasematched for type-II down-conversion; PBS is a polarizing beamsplitter; SPCMs are single photon counters; Pol. is a polarizer. The pump laser is separated from the down-conversion beams using a fused silica prism (not shown). The two-photon state is prepared conditioned on successful postselection of a photon pair after the polarizer. Once prepared in the right quantum state, the light reflects from either a broadband dielectric mirror or a removable interference filter (I.F.) (CVI F10810-1.00-4) (to simulate a medium with a very broad absorption line). The two-photon state is prepared conditionally, when both photons are transmitted through the 45-degree polarizer. This 45-degree polarized light is then split at a polarizing beamsplitter which has a single-photon detector in each output. The PBS acts as a 50/50 beamsplitter on the $45^{\circ}$ polarized photons which allows the pair of SPCMs to act as a two-photon detector.

Fig. 3. Experimental data. The coincidence rate (solid circles) and a singles rate (small open diamonds) as a function of the inter-photon delay are shown in the case where a) the 'absorber' (interference filter) is removed and when b) the 'absorber' is in place.

Fig. 4. Experimental and theoretical normalized ratio of coincidence rates. Plotted is the normalized ratio of the coincidence rates of Fig. 3b to Fig. 3a. The maximum drop in the coincidence rate ratio occurs for delays of $\pm 10 \mathrm{fs}$ and is at least $5 \%$ less than the rate at zero delay. The data have been normalized to the average rate over the delay, $|\tau| \geq 20 \mathrm{fs}$. The theoretical predictions are also shown. The case where there are initially no frequency correlations between the two photons is shown as a solid curve. The case where the frequencies sum to a well-defined value is shown as a dotted curve. 


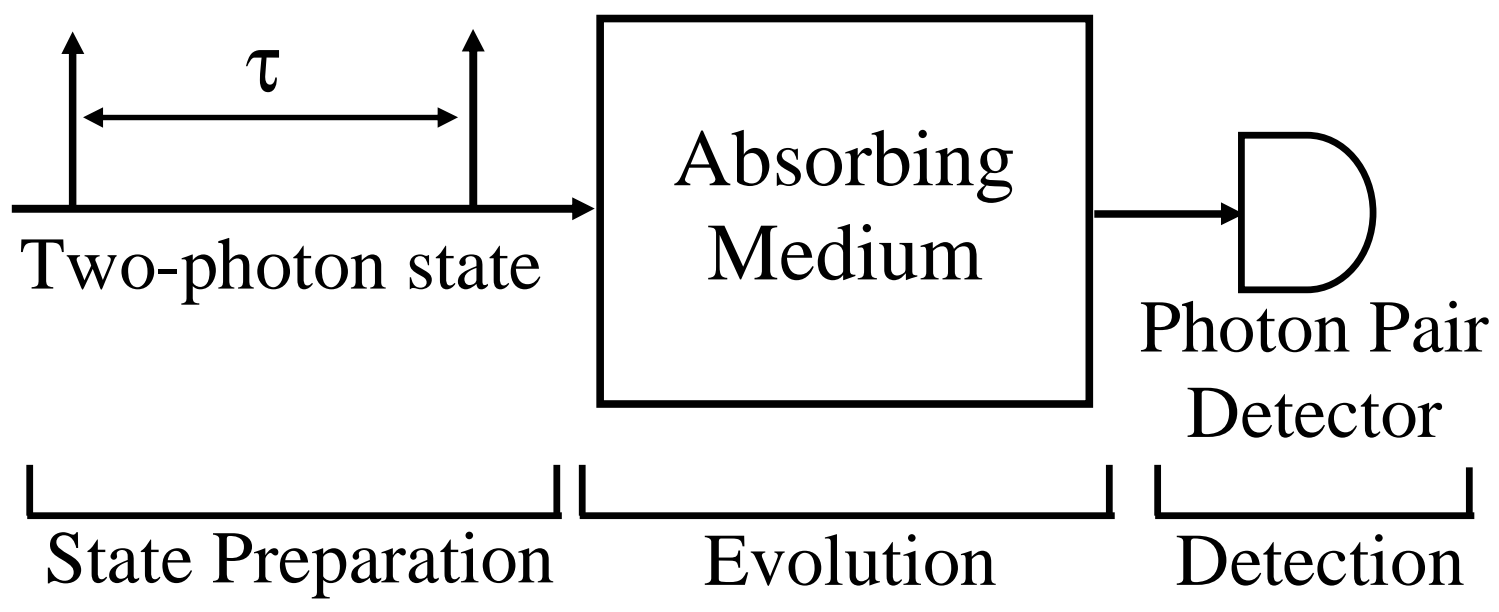




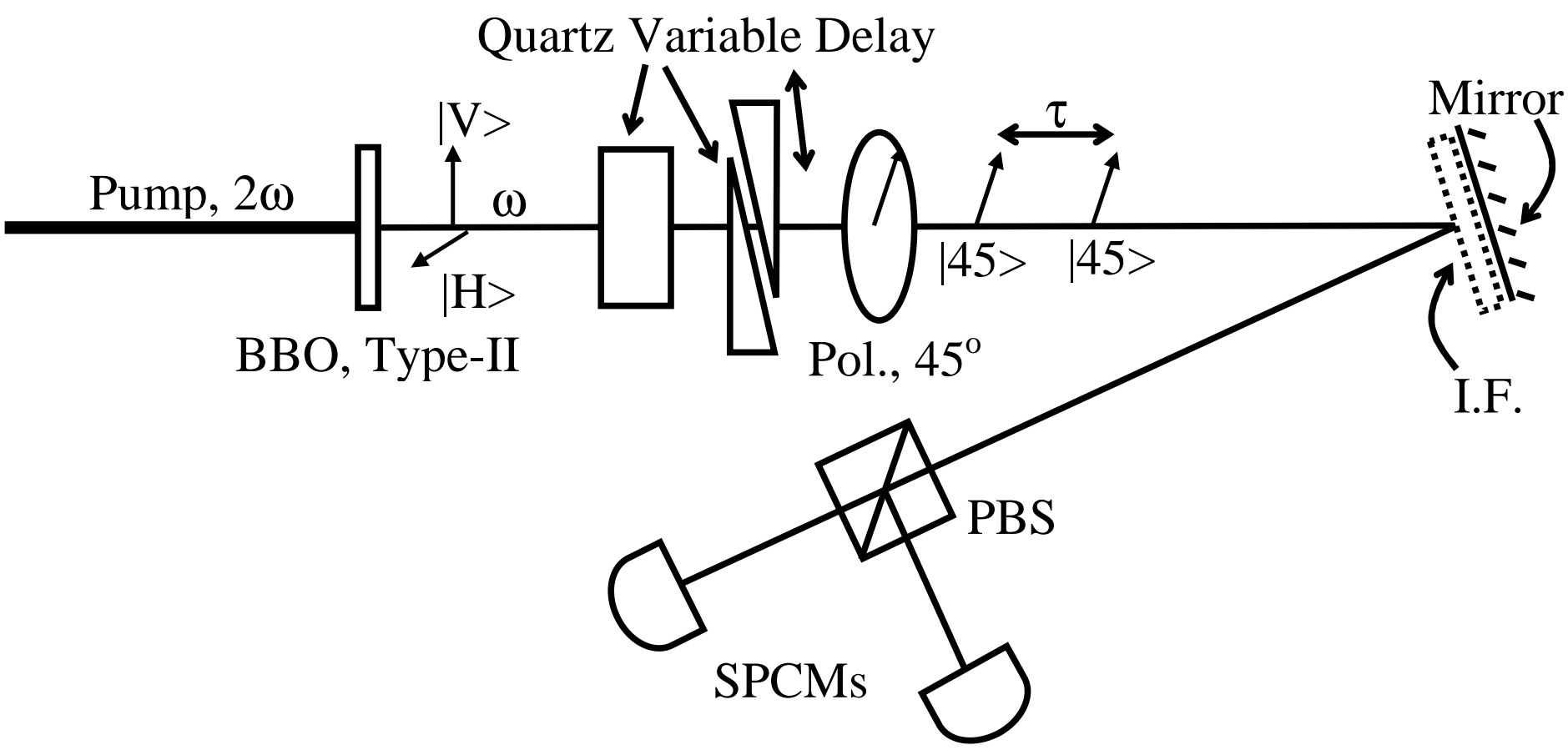




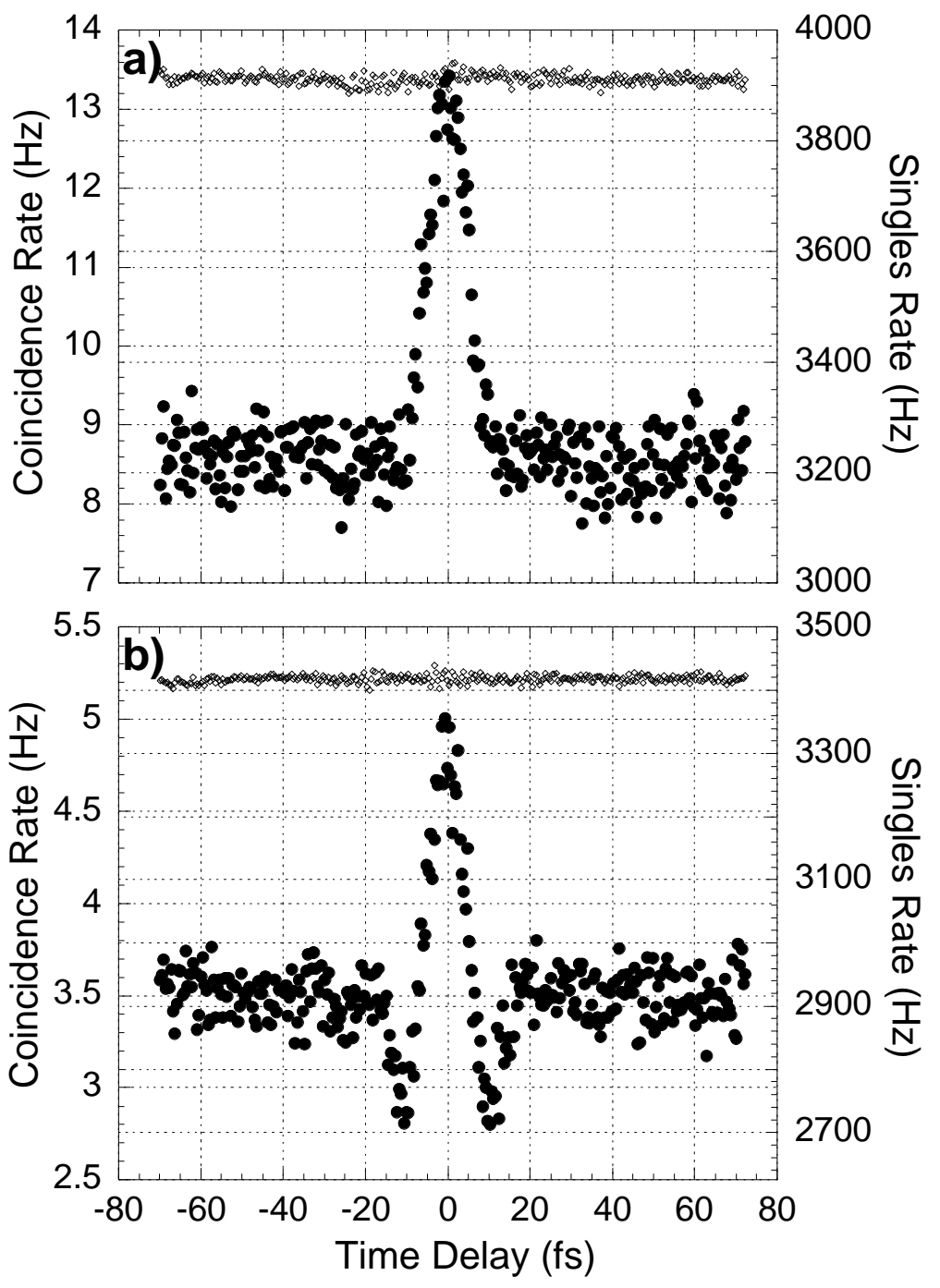




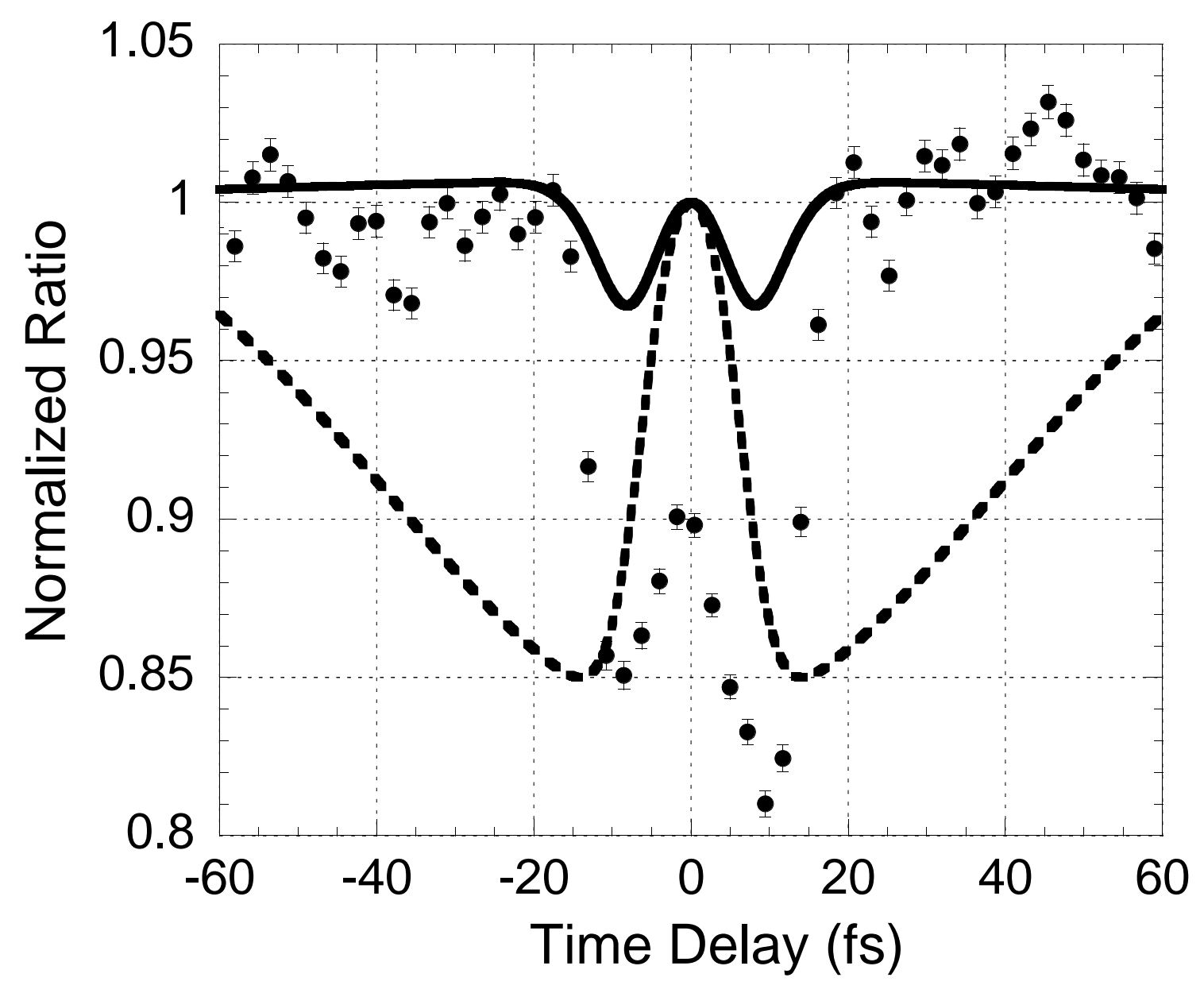

